\title{
THE INVESTIGATION OF A SUSPECTED CASE OF BRAIN TUMOUR.
}

\author{
By C. M. HINDS HOWELL, M.D., F.R.C.P. \\ (Consulting Physician, St. Bartholomew's Hospital; Physician, National Hospital, \\ Queen's Square.)
}

In considering this subject, it seems convenient to discuss briefly the symptoms which are likely to suggest the possible presence of a brain tumour. The early recognition of the presence of such a tumour is of great importance, as its successful removal may depend on this factor. It is, however, necessary to point out that though the appearance of symptoms may be recent, the tumour may have already reached a considerable size; whilst on the other hand symptoms, such as fits, may have existed for a long time, reckoned sometimes in years, before the appearance of signs of increasing intracranial pressure reveals their true nature. This lag in time relationship between the first appearance of a tumour and the first observed symptoms or physical signs is more likely to occur in connection with meningiomata than with other types of brain tumour.

The commoner symptoms which may indicate the presence of a brain tumour may be divided into four main groups:-

(I) Those indicating a general increase in intracranial pressure.

(2) Fits-which may be either focal or generalised.

(3) Changes in personal characteristics.

(4) Focal symptoms, which are likely to be combined with any of the above groups.

The examination of a patient who presents any of the above symptoms must begin with a most careful case history. The taking of such a history is really an art in itself demanding a degree of skill which can only be gained by experience. Whilst taking such a history it is possible to test the patient's power of concentration and expression; his memory, speech, emotional reactions and mental capacity. These must, of course, be correlated with his educational and social status.

The next step is to make a thorough general physical examination of the patient, particularly with regard to his lungs (early bronchial carcinomabronchiectasis), his cardio-vascular system, and blood pressure (hyperpiesis, arterio-sclerosis, polycythæmia, heart block); the abdomen for presence of any tumour, and the rectum, paying particular attention in the male to the condition of the prostate. The urine must, of course, always be examined. Special examinations which may be required in connection with this general overhaul include a blood count and examination of a blood film, blood urea estimation, and possibly X-ray examination of the chest.

- Then follows a complete examination of the nervous system, which naturally includes an ophthalmoscopic examination of the fundus oculi, and taking of the visual fields, at first by simple confrontation tests, supplemented as necessary by perimeter or screen examination. Finally, as part of the examination of the nervous system, a Wassermann reaction must be determined in the blood and cerebro-spinal fluid. When a specimen of the latter is being obtained, the pressure must be determined by manometer. Normal pressures vary approximately from 80 to $160 \mathrm{~m} . \mathrm{m}$. of cerebro-spinal fluid. 
The cerebro-spinal fluid must also be examined for colour, cells, protein qualitatively and quantitatively, and it is advisable to have a Lange's Gold Curve estimation.

I shall refer later to the significance which these findings in the cerebrospinal fluid may have with regard to a positive diagnosis of brain tumour.

Finally a straight X-ray of the skull should be taken. This may be helpful by indicating an increased intracranial pressure owing to the presence of hammer markings, or simple dilatation and thinning of the pituitary fossa, particularly of the posterior clinoid processes. The presence of a meningioma may be suggested by abnormal vascular markings, or infiltration of bone. The position of the pineal body is usually visible, owing to the presence of calcified particles in it, and any lateral displacement may be noted. Abnormal calcification may be seen-perhaps in connection with a tumour or aneurysm of a cerebral artery (Albl rings), or vascular markings with some calcification may show the presence of an angeiomatous condition.

Calcification so common in supra-sellar adenomata will be visible, and the alteration of contour in the pituitary fossa due to pituitary tumour or hydrocephalic ventricular dilatation will also be revealed. It is important to remember that calcified plaques are not uncommon in the falx cerebri under normal conditions.

The patient's symptoms and physical signs, as determined by clinical examination, may now be reviewed.

With regard to the first group, i.e. those which may indicate an increase in intracranial pressure.

Headache, vomiting and papillodema. Of these I think it is generally agreed that headache is the most constant and papillœdema the most likely to be absent.

Headaches due to tumour of the brain may be the first presenting symptom. They may be diffused or localised to one side, in which case the tumour is likely to be situated on the same side as the headache. The headache is usually intermittent, often most marked in the early morning, when it may awaken the patient, or on first getting up. In the terminal stages of brain tumour very intense paroxysms of headache may occur, during which the patient may become semi-conscious. Such paroxysms may be of comparatively short duration. Headaches occur with varying conditions of intracranial pressure. Thus headaches may be severe whilst the intracranial pressure is not raised, and alternatively the intracranial pressure may be high, whilst headache is not a prominent symptom.

Tumours in the posterior fossa usually give rise to occipital headache, but it is important to remember that some patients suffering from hypertension are troubled with severe occipital headache. Cases also occur in which the patient complains at intervals of severe headache associated with vomiting and prostration. A diagnosis of migraine may thus be suggested, for the patient, between the attacks, may be quite free from symptoms. The development of papillodema will, of course, negative the diagnosis of migraine, or some abnormal physical sign such as an extensor plantar reflex will show the presence of a 
gross cerebral disorder. In such cases a ventriculogram is likely to show dilated cerebral ventricles, indicating the presence of hydrocephalus, which in a number of cases is not the result of tumour. Indeed it is possible that the cause of the hydrocephalus is revealed neither by operation, nor post mortem examination. Following infections of the middle ear, the patient may develop severe headache, papillœdema and possibly sixth nerve palsy, either single or bilateral. Lumbar puncture may reveal a fluid under great pressure but otherwise normal. Symonds has described such cases under the title of otitic hydrocephalus. Their recognition is a matter of very great importance, as the symptoms suggest the possibility of brain abscess. The normal cerebro-spinal fluid will exclude this, and the cases recover spontaneously, such recovery being accelerated by repeated lumbar puncture.

Papilloedema due to tumour may be more marked in one eye than the other, but this is not a reliable guide, as Horsley thought, to the side of the brain on which the tumour is situated. It is, of course, most important to distinguish the swelling of the disc which occurs in some cases of hypertension, from that due to tumour. In the former cases hæmorrhages are always a very marked feature, whilst the greatly raised blood pressure and the condition of the retinal arteries serves further to differentiate the two conditions.

Papillodema in one eye with primary optic atrophy in the other is a characteristic picture particularly in meningiomata lying on the orbital surface of one frontal lobe. The optic atrophy is, of course, due to direct pressure from the tumour on the optic nerve. Papillodema, associated with pain in or behind one or both eyes may occur as the result of retrobulbar neuritis.

Vomiting is a symptom common enough in conditions of increased intracranial pressure. It is liable to be sudden and unaccompanied by nausea. I have already drawn attention to the possibility of such vomiting, associated with severe intermittent headache, being regarded as a manifestation of migraine, but it is unusual in cases of cerebral tumour to have any prolonged interval of seemingly normal health between the bouts of headache and vomiting. I have, however, met with this condition myself in several cases of hydrocephalic dilatation of the ventricles, apart from the presence of a tumour.

Arterial hypertension may be a cause of severe headache and vomiting either in association with hypertensive cerebral attacks or with uræmia-both of which conditions must be excluded. I do not think it is possible to distinguish with any certainty what is called "cerebral vomiting" from that due to other causes, except in so far as the possible absence of nausea in cerebral cases may be of some assistance.

With regard to the second symptom on my list, namely, Fits; these are of common occurrence in cases of intracranial tumour, and in an adult particularly should suggest the probability of the fits being symptomatic rather than "idiopathic."

In cases of cerebral tumour, the fit may assume a Jacksonian type, in which case careful observation of the attack will be of definite value in localising the situation of the tumour.

But in many cases the fits are not focal but generalised in character. My impression, unsupported by statistical evidence, is that the generalised are more usual than the focal type of convulsion. I agree with Walshe's view that fits due to intracranial tumour are rarely nocturnal. 
In the early stages of tumour, particularly I think with infiltrating gliomata, the attacks may be of a quite temporary inhibitory type, such as transient aphasia,, or transient loss of power in a limb, without any loss or disturbance of conscious-.ness, and without any convulsive movement.

I turn next to the third group of symptoms-Changes in personalo characteristics. These may be either mental or physical.

Mental changes may result from a tumour in any situation, but are probably earlier in appearance and more pronounced when the tumour is situated in the prefrontal region. In one personally observed case, the first symptom to cause any comment was the patient's bathing in the sea with his tobacco pouch firmly: grasped in his hand.

Lack of initiative and apathy appearing in an individual whose previous characteristics have been quite the contrary, or the development of a good?. natured fatuity (Witzelsucht) are also common symptoms of tumour in the frontal $\vec{r}$ lobes, as is also the disregard of the usually accepted standards of polite $\overrightarrow{ }$ behaviour.

It is important to note that these personality changes may precede the $\vec{\nexists}$ development of any of the more obvious signs of increased intracranial pressure. 9

The physical changes to which I have referred arise as a rule from disturb- $\vec{z}$ ance of normal functions of the pituitary gland or the hypothalamus, for a detailed description of which the reader is referred to the appropriate sections of $\overrightarrow{0}$ this publication, where will be found also the descriptions of the focal symptongse to which I have referred as the fourth of the common symptom groups of bran. tumour.

Having decided that the symptoms of which the patient complains, taken in conjunction with the physical signs, or in the absence of any definite clinicalo findings, suggest the possibility of a brain tumour, we have now to consider what further steps can be taken to confirm the diagnosis, and to localise the siteo of such a tumour, if present. I propose to discuss briefly the methods that are in use at the present time for this purpose, and shall consider them under four headings, namely, lumbar puncture, encephalography, ventriculography and arteriography.

\section{Lumbar Puncture.}

(a) Pressure. In all cases the pressure of the cerebro-spinal fluid must be measured by manometer and the cerebro-spinal fluid examined cytologically, chemically, and for the Wassermann reaction. A Lange's gold curve should also be included in the examination.

Where it is certain that a brain tumour is present, lumbar puncture must be done with caution, and only a very small volume of fluid removed, if the intracranial pressure is found to be greatly raised.

Contrary to what has been the commonly accepted view, it seems probable? that lumbar, and ventricular punctures are potentially more dangerous in supra $\frac{10}{5}$ tentorial than in subtentorial tumours.

In supratentorial tumours with greatly increased intracranial pressure there is a tendency to herniation of the temporal lobe through the tentorium; and this ${ }_{\mathbb{N}}^{\circ}$ tendency is, no doubt, aggravated by lumbar puncture. 
Smyth and Henderson have found that in a certain proportion of such cases the intraventricular pressure is greater than the lumbar pressure, and further that whilst jugular compression causes a rapid rise in intraventricular pressure, the rise in lumbar pressure in these cases takes place slowly and to a lesser extent. This was so in 8 out of 33 cases of brain tumour, which they investigated in this way at the National Hospital.

In cases of subtentorial tumour, the intraventricular and lumbar pressures were identical, and the variation of pressures on jugular compression the same.

Where the ventricular pressure is higher than the lumbar pressure, the prognosis is so much the more serious, and the ventricular tap should be followed by immediate operation.

The normal pressure of cerebro-spinal fluid as measured by lumbar puncture varies from about 80 or $90 \mathrm{~mm}$. of water to $160 \mathrm{~mm}$. Any pressure above the latter figure may be judged to indicate a raised intracranial pressure.

In the great majority of cases of brain tumour, the lumbar cerebro-spinal fluid pressure is definitely raised. Smyth and Henderson in their series of 33 cases found 5 with pressures either within normal limits or not much raised; the highest reading in this group was $185 \mathrm{~mm}$. (the patient had a meningioma of the left parietal cortex weighing I32 gr.); the lowest pressure recorded was $90 \mathrm{~mm}$. (right temporal cystic glioma.)

From which the important fact emerges that a brain tumour may exist with a normal or even low pressure in the cerebro-spinal fluid as measured by lumbar puncture. Nevertheless in a doubtful case the presence of a definitely increased intracranial pressure is a fact of great importance. In connection with the question of pressures of the cerebro-spinal fluid I should perhaps mention what is known as Ayala's quotient. By this is meant the ratio of the fall in pressure in the cerebro-spinal fluid (after removing some of the fluid) to the volume of fluid removed. Quoted as a fraction the formula is:

$$
A=\frac{Q \times F}{I} \quad \text { where } \begin{aligned}
& Q=\text { amount of fluid withdrawn. } \\
& \begin{array}{l}
F \\
I=\text { final pressure. } \\
\mathrm{I}=\text { initial pressure. }
\end{array}
\end{aligned}
$$

I do not think this quotient is of much practical value, but quotients lower than 5 suggest the presence of tumour, and above 7 serous meningitis.

(b) Cytology. In brain tumour the number of cells in the cerebro-spinal fluid is not usually increased above the normal figure, but occasionally there is such an increase, which may include the presence of tumour cells. It is in cases where the tumour is in direct contact with the cerebro-spinal fluid either within the ventricles or on the surface of the brain that a cellular increase may be looked for. But even in these circumstances the histological structure of the tumour is of significance in relation to the cellular increase-Greenfield and Carmichael found no increase in cells in 9 cases of endothelioma, whilst in 27 cases of glioma cerebri 6 showed an increase in cells varying from to to 79 per c.mm., in all of which the tumour had reached the ventricular wall. With pituitary and supra sellar tumours there may also be found some increase in the number of cells in the cerebro-spinal fluid (lymphocytes). 
(c) Protein. What has been said about the cytology of the cerebro-spinal $\frac{a}{2}$ fluid in cases of brain tumour may be repeated with regard to protein. In the majority of cases there is no increase above the normal figure of 20 to $40 \mathrm{mgm}$... per cent. But where the tumour is a vascular one and in direct contact with the cerebro-spinal fluid an increase in its protein content may be expected.

Rather unexpectedly neurofibromata, the most common occurrence of which is in connection with the auditory nerve, usually give rise to an increased protein content in the cerebro-spinal fluid without any corresponding cellular increase. Such protein increase may be considerable. In a case mentioned by Greenfield and Catrinichael the figure of I75 mgm. per Ioo c.c. was recorded.

(d) The Wassermann reaction. The Wassermann reaction is obviously of $\vec{c}$ great importance in connection with the examination of the cerebro-spinal fluid and the Lange gold curve may be of considerable value as well. In a certain number of cases the differential diagnosis of disseminated sclerosis from brain tumour may present considerable difficulty. So far as the cerebro-spinal fluid is concerned in both cases the pressure is likely to be raised, though in disseminated sclerosis rarely above $200 \mathrm{~mm}$. In both there may be some increase in protein and cells. But in cases of disseminated sclerosis perhaps a third will show a paretic type of Lange curve, whilst I have never seen such a curve in 9 cases of brain tumour. A paretic type of curve with a negative Wassermann reaction will suggest strongly the diagnosis of disseminated sclerosis.

\section{Encephalography and Ventriculography.}

The introduction of air either by the spinal route (encephalography) directly into the ventricles (ventriculography) has proved to be a procedure enormaus importance in connection with the diagnosis, localization, and, in consequence, the successful surgical treatment of brain tumours. The method is one which is by no means devoid of risk. It must be used as complementary to a full physical examination and certainly not allowed to replace such an examination; and in many cases, particularly of ventriculography, the surgeon must be ready to proceed with the operation without delay.

(I) Encephalography. This method should not be employed when the pressure of the cerebro-spinal fluid is definitely raised above the normal maximum figure-I60 to $I 70 \mathrm{~mm}$.; nor when considerable papillodema is present. The amount of air to be introduced varies with the predilection of different authorities. I am accustomed to using 40 c.c.'s of air, which will I think usually be found adequate. The air is introduced by the normal lumbar puncture route with the patient in the sitting position; II c.c.'s of cerebro-spinal fluid are withdrawn and Io c.c.'s of air introduced slowly. This is repeated until approximately 40 c.c.'s of air have been introduced. About Io per cent. less air is used than the volume of cerebro-spinal fluid withdrawn to allow for the expansion of air at body temperature. X-ray photographs will as a rule show air in the ventricles and also distributed over the cortex and in the basal cisternæ. Severe headache is likely to follow the injection of air by this route and will require control, as for example by sodium amytal gr. 3 , followed if necessary by veganin tablets or some similar preparation.

The indication for encephalography, rather than ventriculography, in connection with suspected brain tumour would be the absence of papillodema, the absence of unduly raised intracranial pressure, or the occurrence of epileptic attacks which might suggest the presence of brain tumour. 
As an illustration of the use of encephalography for the purpose of excluding brain tumour, I might mention the case of a man aged 23 under my care at the National Hospital, who had suffered from infrequent epileptic attacks for two years. Nothing abnormal was discovered on examination, except that the cerebrospinal fluid had a high "normal" pressure figure, and that there were $240 \mathrm{mgm}$. protein present in it. Although this is a very high figure, as we have seen, for a case of brain tumour, I thought it advisable to have an encephalography made. The resulting picture appeared quite normal.

Encephalography will not, of course, give such reliable information as will ventriculography as to the condition of the ventricles, because the filling of these is not so certain, and thus the pressure of a tumour which is certainly present may not be shown by this method. For example, a man of 37 under my care had complained for four months of attacks of "pins and needles" down the whole of the left leg from groin to the toe, the attacks lasting about two minutes. About the same time he noticed that the left ankle felt weak after walking for some distance. He had not complained of any headache, or indeed of any other symptom. He had no papilloedema. There was definite loss of power in the left foot with increased tone in the left leg, increased knee jerk and extensor plantar on the left side_. The left abdominal reflex was absent; cerebro-spinal fluid pressure $\mathrm{I} 65 \mathrm{~mm}$.; no cells were seen; protein $20 \mathrm{mgm}$.; Wassermann reaction negative in blood and cerebro-spinal fluid Lange curve no change. An encephalograph showed the sulci well filled, but air had not entered the ventricles. The character of the attacks of paræsthesia, followed by weakness in the left foot suggested a focal brain lesion rather than disseminated sclerosis. At operation a right sided parasagittal meningioma was found and successfully removed by $\mathrm{Mr}$. Harvey Jackson.

(2) Ventriculography. This method was introduced by Dandy in I9I8, since which date its use has progressively increased, to the point where it seems that some neurological surgeons practise it to the exclusion of clinical examination. To such practise the most strenuous opposition should be offered. The method is not devoid of risk to life, the mortality rate varying in the hands of different surgeons with an average of 3 per cent., but rising in some cases to the remarkable figure of I3 per cent., a figure which suggests that this method was employed in a number of cases without regard to the clinical condition of the patient.

In late cases with presumably extensive tumours causing a great increase in intracranial pressure with marked papillodema, the method should not be employed. Its chief use should be restricted to early cases in which there is no certain indication as the result of the clinical examination as to the site of the tumour; but at least its dangers should be weighed against any possible advantage that might accrue from its use.

Quite apart from risk to life, not a few patients experience severe and distressing after effects unless operation is carried out immediately following the taking of X-ray pictures. Wherever possible this course should be adopted. The symptoms to which it may give rise include headache (though this is often less severe than in cases of encephalography), drowsiness, vomiting, sometimes convulsions, and some degree of pyrexia.

The amount of air to be introduced will depend on the amount of cerebrospinal fluid removed, and should as a rule be about 5 to ro per cent. less than this. 
Though I have repeatedly stressed the importance of a thorough clinical examination in all cases of suspected brain tumour, it is quite certain that in not a few cases where the diagnosis of tumour is certain, exact localization is impossible. An instructive illustration of this is the following:-

A. B., aged 36, a casual labourer, was admitted under my care at the National Hospital with a history of headaches for 12 months and failing vision for two months. Severe headaches had recurred at repeated intervals of two or three days and had been chiefly occipital in situation. He sometimes had lights in front of the eyes, a pinkish colour with zigzags, seen in both eyes. The patient was dull, retarded, and apathetic. There was papillœdema in both eyes, no weakness in the arms, but he thought that both legs were weaker than normal. He was found to have pupils which did not contract to light or convergence, an absent left abdominal reflex and extensor plantar reflex on the left side. Before his admission to hospital he had been seen by a distinguished neurological surgeon, who having no vacancy sent him to Queen Square with the diagnosis of tumour of the right hemisphere spreading to midbrain.

On admission to Queen Square, the man was in a very drowsy condition and no very clear statement could be obtained from him. We found that he had the eye signs already referred to; the abdominal reflexes were diminished on the left side, as was also the knee jerk. The left plantar reflex was a doubtful extensor. An additional sign that was then elicited was a rather coarse tremor in the outstretched right arm.

As these signs were equivocal Mr. Harvey Jackson filled the ventricles with air. The anterior horn of the left lateral ventricle did not fill, and the ventricular system was displaced to right. As a result of the ventriculogram a tumour was located in the left frontal lobe. Mr. Harvey Jackson removed a meningiomas weighing I70 grs. measuring $6.5 \mathrm{cms} . \times 9.3 \mathrm{cms}$. A straight X-ray had shown no응 abnormality in this area. The man made a good recovery.

\section{Arteriography.}

A radio opaque substance is injected into the carotid artery and $\mathrm{X}$-ray photographs are taken immediately this has been done, and directly afterwards.

Sodium iodide was used at first by Morris in I927. This was, however, found to produce undesirable results, such as fits, pain, etc., and a 25 per cent. colloidal suspension of thorium dioxide, known as thorotrast, is now used. This substance has slight radio active properties. There are those who believe, as the result of laboratory experiments, that it is likely to have ultimately harmful effects on the human body. Others have found in practice no such results; but the question cannot be regarded as finally settled. The use of thorotrast is not free from immediate danger. A patient of mine received Io c.c. into the carotid artery and immediately complained of severe pain in one eye, with loss of sight. The eye became proptosed, the conjunctiva intensely inflamed. Ophthalmoscopic examination revealed a green impenetrable screen of some kind. After three months the eye recovered completely. I know of another similar case, and of at least one case of sudden death. On the other hand, thousands of injections of thorotrast must have been done by now with no inconvenience to the patient.

The chief use of this method is to demonstrate the presence or absence of aneurysm, or of an angeiomatous tumour. It is possible that in a few cases it might be of assistance to the surgeon in planning his operation to know of any deviation from normal in the distribution of the larger ventricles. 
From the foregoing necessarily brief account of the various measures which may be employed in proving the presence and locating the site of a brain tumour, it will be apparent that much progress has been made within a comparatively short time. No doubt it is to be expected that further progress will be made in the future.

What is to be done when such a tumour has been diagnosed and its situation more or less accurately located is a matter of much greater difficulty than the diagnosis. The question to be decided is whether removal of the tumour should be attempted, or treatment by physical methods (X-ray or radium) or whether it is best to adopt merely palliative measures. Much will depend on the circumstances as revealed by the history of the case, the condition of the patient, and the probable nature of the tumour. It is fair, I think, to say that at the present time there are two view points with regard to procedure-one, realising that the patient is doomed if nothing is done would urge operative treatment in practically every case, since once in a while, even in the most discouraging circumstances, the seemingly impossible happens and the patient is rescued. The other view, more in accord with the practice of general surgery, is to operate only when there seems a reasonable hope that such operation will be successful.

Our aim must be to improve our means of arriving at more exact information of conditions within the skull at as early a stage as possible, or to discover " a cure for brain tumour" without operation. 